\title{
PERFORMANCE ANALYSIS OF MOBILE AD- HOC NETWORK ROUTING PROTOCOLS OVER TCP
}

\author{
Rahat Hossain Faisal ${ }^{1}$, A. N. M. Tawhidul Islam ${ }^{2}$, Md. Sadek Ali ${ }^{3}$, Farruk Ahmed ${ }^{4}$ \\ ${ }^{1}$ Department of Electronics and Telecommunication Engineering, Prime University, Dhaka, \\ Bangladesh \\ rhfaisaleymail.com \\ ${ }^{2}$ Department of CSE, Islamic University, Kushtia, Bangladesh \\ tawhideibtra.com \\ ${ }^{3}$ Dept. of ICE, Islamic University, Kushtia, Bangladesh \\ sadek_ice@yahoo.com
}

${ }^{4}$ School of Engineering and Computer Science, Independent University, Dhaka, Bangladesh.

farrukesecs.iub.edu.bd

\begin{abstract}
In order to reduce the communication cost and time we are looking forward for successful implementation of an infrastructure less network like Mobile Ad Hoc Network in all arena of wireless mobile communication. But still it is a challenge to decide a most appropriate routing protocol for MANET. In MANET there is no fixed topology due to the mobility of nodes, interference, multipath propagation and path loss. Since MANET does not use fixed infrastructure rather it always have to find suitable router and routing path for each communication, existing established routing protocol is not suitable for MANET to function properly. Different Routing protocols have been proposed to meet the challenges with MANETs. This paper evaluates the performances of four MANET routing protocols which are DSDV, AODV, DSR and TORA over TCP, a Transport Layer Protocol. The performance metrics which are considered in this paper are packet delivery fractions, normalized routing overload, end to end delay and throughput. DSDV is a proactive protocol and the others are reactive protocols.
\end{abstract}

\section{KEYWORDS}

MANET, TCP, Routing Protocols, Wireless, QoS.

\section{INTRODUCTION}

A wireless mobile ad-hoc network consists of mobile nodes that are interconnected by wireless-multi-hop communication paths. The nodes and networks are independent and they are controlled by themselves as per requirement. Figure1 depicts a sample MANET. 


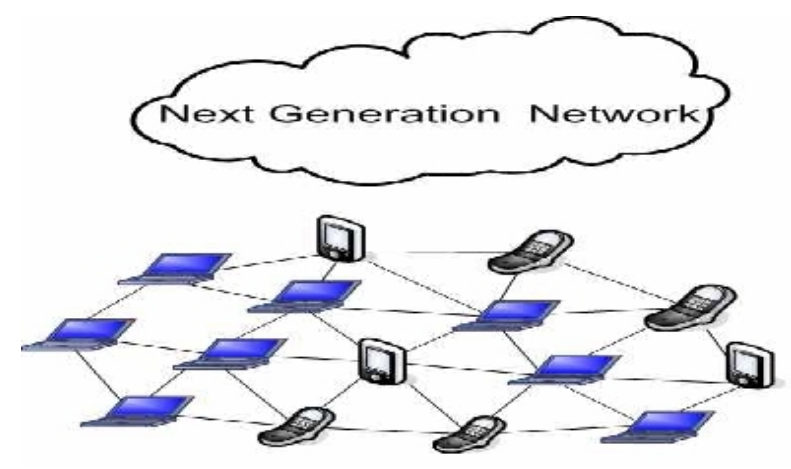

Figure 1. Mobile Ad Hoc Network [6]

These mobile ad-hoc networks offer unique benefits and versatility for certain environments and applications. In MANET at the same time the mobile nodes are used as transmitter, receiver and router, so no extra base station is required. It can be deployed immediately and at any terrain. Since all nodes are allowed to be mobile, the topology of such networks is necessarily time varying. Thus these types of networks offer many advantages where settings up wired line networks are not feasible. Such advantages attracted immediate interest in its early use among military, police, and rescue agencies, and especially under disorganized or hostile environments, including isolated scenes of natural disaster and armed conflict.

\section{MANets Routing Protocols}

The major challenges that a routing protocol designed for MANETs faces are [1]:

1. Mobility,

2. Bandwidth constraint,

3. Error-Prone Shared Broadcast Radio Channel

4. Hidden and Exposed Terminal Problems

5. Resource Constraints

Due to these challenges in MANETs environment wired network routing protocols cannot be used. So special routing protocol is required which will perfectly fit with MANETs environment for successful and precise communication through MANETs. Different types of routing protocols for MANETs are proposed which can be categorised in different ways such as according to Routing Information Update Mechanism, according to use of Temporal Information for Routing, according to the Topology Information Organization, and it can also be classified according to Utilization of Specific Resources. Some proposed MANETs routing protocols are mentioned bellow [1]: 


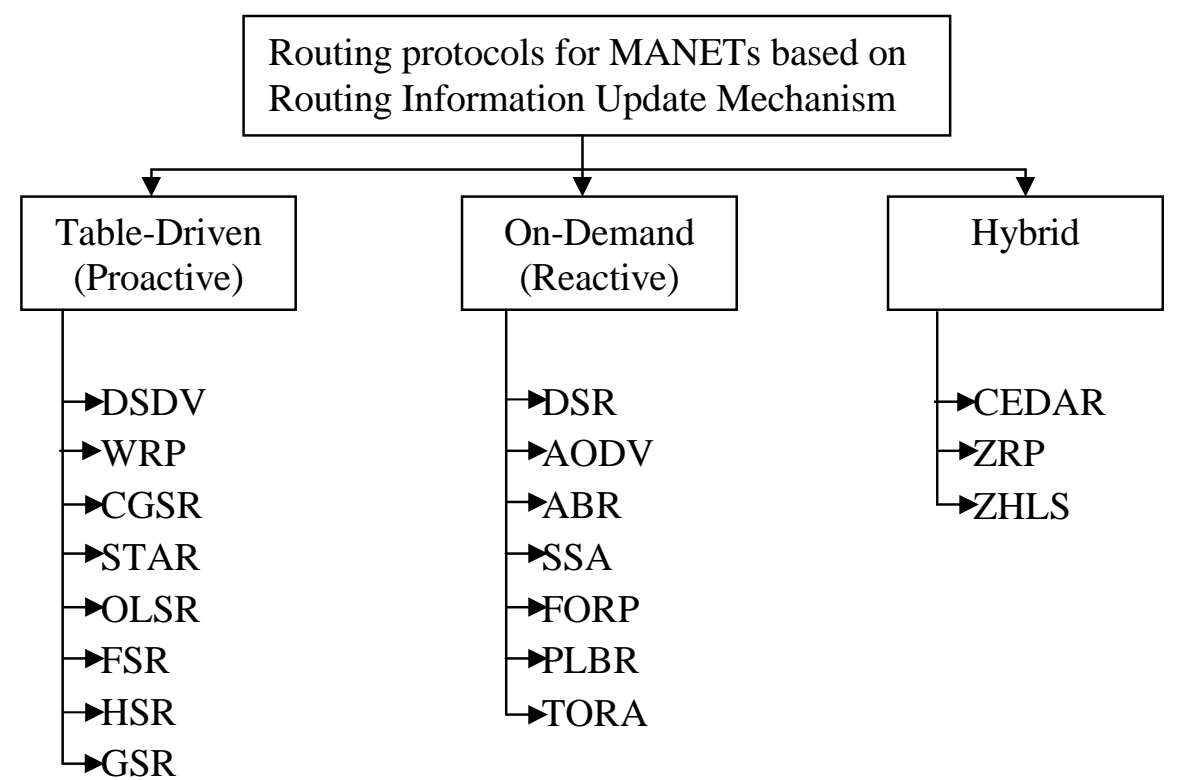

Figure 2. MANET Routing Protocols

Yet, it is a major challenge to find the most appropriate routing protocol for MANETs. A routing protocol for mobile ad-hoc wireless network should have the following characteristics [1]:

1. It should be fully distributed.

2. It must be adaptive to frequent topology changes.

3. Minimum connection setup time is desired.

4. It must be localised.

5. It should be loop-free and free from stale routs.

6. Number of broadcasts made by each node should be limited to reduce packet collision.

7. It must converge to the optimal routes quickly once the network topology becomes stable.

8. It must optimally use scarce resources such as bandwidth, computing power, memory, and battery power.

9.Every node in the network should try to store information regarding the stable local topology only.

10.Certain level of QoS should be maintained as demanded by the applications, and timesensitive traffic should be supported.

Considering the challenges and expected characteristics for MANET routing protocol we have considered the AODV, DSR, TORA and DSDV for our present research paper to analyze their performances on different performance metrics. In those cases where a protocol supports both unicast and multicast routing we have only considered the unicast routing part. Research on AODV, DSR, TORA and DSDV is done over two transport layer protocols, TCP and UDP. In this paper we have only presented the performances metrics over TCP.

\subsection{Destination Sequenced Distance-Vector (DSDV) Routing}

DSDV [3] maintains a distance vector table of mobile node to mobile node like distance vector routing protocol. Each node contains a routing table for all reachable destinations which stores the next-hop and number of hops for the destination. Routing updates are periodically broadcasted by each node in DSDV. The advantage with DSDV over traditional distance vector protocols is that DSDV guarantees loop-freedom through using sequence numbers to tag each route. 
International Journal on AdHoc Networking Systems (IJANS) Vol. 2, No. 3, July 2012

\subsection{Ad Hoc On-Demand Distance-Vector (AODV) Routing}

AODV [6] protocol is reactive protocol, which find a route to destination on demand whenever communication is needed. Whenever it defines a route for a certain communication it tries to reduce extra load on the route. The algorithm does not consider the location of the nodes rather it uses a table to find the optimum route and sequence numbers is used for the destinations to route the packets to the destination mobile node. A source sends data only when it is requested. Route request (RREQ) packets are broadcasted within the network whenever a source demanded a communication to be established with the destination mobile node. When a RREQ is received by a mobile node, it checks it's routing table and decide whether he himself is the destination node or he has a route to the destination within his table. According to his finding in his table it unicast a route reply (RREP) to the sources according to the temporary reverse route generated by the source node or it floods RREQ again within the network.

\subsection{Dynamic Source Routing (DSR)}

DSR [4] is a distance vector routing protocol that makes use of sequence numbers to avoid routing loops. However, it is a reactive algorithm and as such does not maintain routes to all possible destinations but establishes them as the need arises. Each intermediate node does not need to contain up-to-date information for a complete path to a destination because the complete route a packet must follow to reach its destination which is imprinted on its header by the source. DSR makes extensive use of route caching and as such its table entries may contain multiple routes for the same destination. Furthermore, there is no need for a mechanism to detect routing loops as loop freedom is assured by source routing.

In order for the source to discover the path to a destination the network is controllably flooded with Route Request (RREQ) packets. As a RREQ packet is rebroadcast by the intermediate nodes, the hop sequence to the destination is recorded on the packet's header. When the packet reaches the destination or a node that knows the route to the destination, a Route Reply (RREP) is transmitted back to the source by reversing the path of the RREQ packet, thus informing the source of the new route. In the case of a unidirectional link, it is necessary for the destination to initiate its own route discovery process as the inverse of the original path is not a valid path in itself.

\subsection{Temporally Ordered Routing (TORA)}

TORA [5] is a distributed routing protocol. It is a link reversal algorithm. It also reduces the reactions to the changes of topology. A key concept in its design is that control messages are typically localized to a very small set of nodes. It guarantees that all routes are loop-free (temporary loops may form), and typically provides multiple routes for any source/destination pair. TORA defines the route for the communication but it uses Internet MANET Encapsulation Protocol for basic functions for communication.

A height value is assigned to each and every mobile node within the network. In TORA, movement of the control data/packets is from higher height node to a lower height node. Query (QRY) and Update (UPD) messages are used to locate routers. A source node broadcasts a QRY packet when it wants to communicate with a destination node. QRY message travels throughout the network to find the destination node or a node which contains a further fresh route. At this stage, the discovered node floods a UPD messages which contains the height of the node. The node which receives this UPD packet set its own height to a higher value. The node will then broadcast its own UPD packet. Thus this process will return many directed links from the source to the destination. That is this process can result in multiple routes. Invalid routes are erased using clear (CLR) messages. 
International Journal on AdHoc Networking Systems (IJANS) Vol. 2, No. 3, July 2012

\section{Simulation Methodology}

For simulation NS2 is used and the following parameters are considered:

\subsection{Simulation Topography}

For network simulations $500 \times 500$ meter flat grid topography is used since the square topography provides a more rigorous environment for performance comparison.

\subsection{Traffic Model}

For all our simulations, we have used the same traffic model that is of $2,4,8$ and 16 FTP sources, transmitting at 4 packets per second with the size of each data packet being fixed at 1024 bytes, because of larger sized data packets lead to higher packet drops due to queuing delay and these considerations have taken for TCP, transport layer protocol.

\subsection{Mobility Pattern and Movement Scenarios}

The setdest utility of NS2 is used to generate the random mobility pattern of the nodes for our simulations. Setdest, uses the random waypoint model to generate random mobility. At the start of simulation, all the nodes are laid out randomly on the rectangular topography and then start moving towards a random destination at some random speed, which is a value between 0 and the maximum specified speed. After reaching the destination, the nodes start to move towards the next randomly chosen destination. The speed however doesn't change when the nodes are mid way to destination.

For random mobility we chose to simulate at five speeds i.e. of $4 \mathrm{~m} / \mathrm{s}, 8 \mathrm{~m} / \mathrm{s}, 12 \mathrm{~m} / \mathrm{s}, 16 \mathrm{~m} / \mathrm{s}$ and $20 \mathrm{~m} / \mathrm{s}$. We also simulated with four different numbers of nodes i.e. 4, 8, 16 and 32 nodes at the above speeds. In this paper we have presented the simulation for 32 nodes.

Each movement simulation lasted for a period of 200s. Since the protocols are sensitive to movement pattern and relative placement of nodes on the topography, we generated 10 different movement scenarios for each simulation and the average of ten simulations were used for collecting data points for presenting the results.

For each line in the graph, we have done five simulations and for each graph we have done twenty simulations.

\section{Performance Metrics}

\subsection{Packet Delivery Fraction}

When FTP is concern, then the ratio of the number of packets originated from the source through its application layer and the number of packets received by destination is defined as packet delivery function (PDF). This metric describes the loss rate which will be found by the transport protocols. Thus packet delivery fraction in turn reflects the maximum throughput that the network can support. It also can be described in terms of data loss rate.

\subsection{Normalized Routing Overload}

Normalized Routing Overhead (NRO) defines the average total number of routing packets transmitted during the simulation. The concern packets are the packets which are sent through multiple nodes and transmission through one hop is considered as one transmission. 
Normalized routing Overload can be computed either as a count of the total bytes sent out as routing packets or a count of total number of routing packets sent out. Here we have used the number of routing packets as our metric because, as we claim that the routing algorithms suppresses the unnecessary periodic updates sent out by nodes, so we needed a measure of how many routing packets were saved in a specific routing protocol and what is its roll in TCP. With more routing Overload, the amount of delay experienced by data packets in reaching their destination would be more as there would be congestion, collision and queuing delay. But now as we are reducing the routing overload, it naturally would lead to better packet delivery times.

\subsection{End to End Delay}

The End to end delay is the average time difference between the moments when packet received at IP layer at the source node and the moment when the packet received at the IP layer of the destination node. End to end delay need to be measured to show its affect against various protocols. For better performance in MANET the end to end delay is required to be reduced as much as possible.

\subsection{Throughput}

The number of received packets per unit of time is known as the throughput and the hroughput should be as higher.

\section{RESUlts AND ANALYSIS}

\subsection{Packet Delivery Fraction (PDF)}

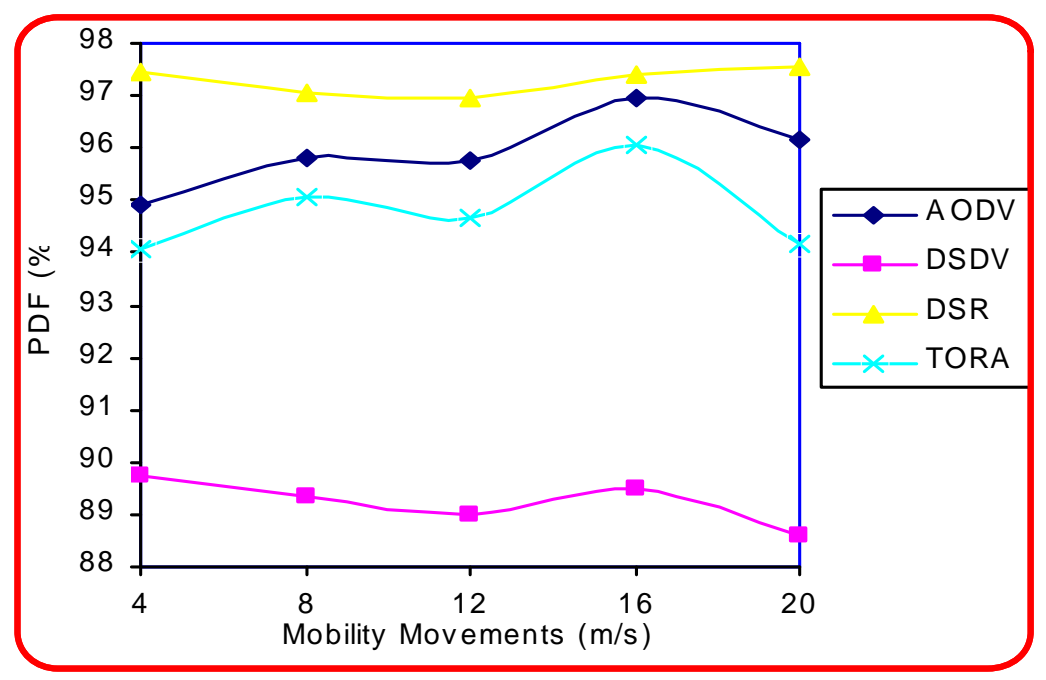

Figure 3. PDF for 32 nodes.

From the above plots we found that the DSDV's performance is the lowest and DSR's performance is the highest in terms of PDF. Here, the performance of TORA is better than AODV but AODV performs much better than DSDV. DSR's performance is almost same in various movement speeds of the nodes but other protocols performance varies according to the change of mobility movements. 


\subsection{Normalized Routing Overload (NRO)}

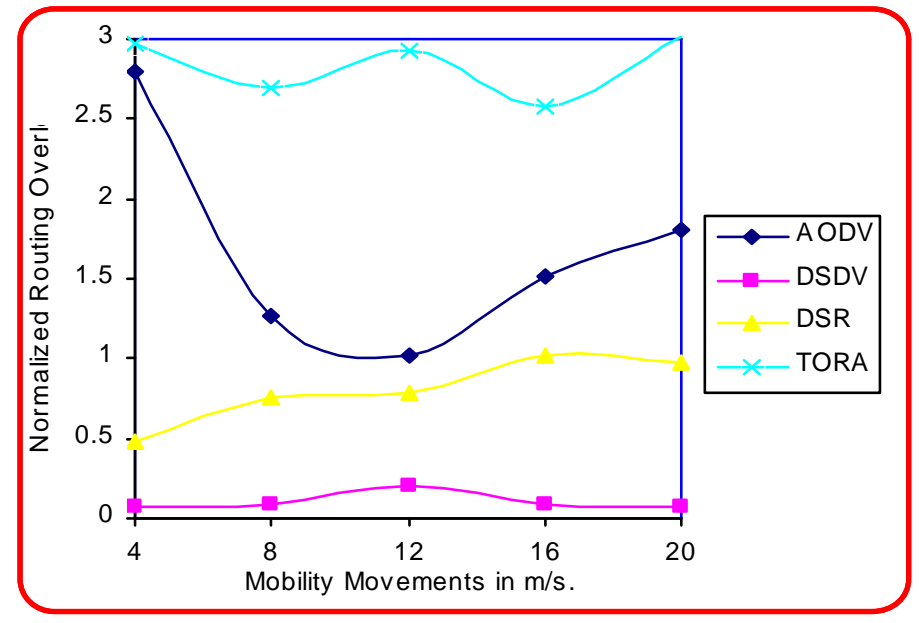

Figure 4. Normalized Routing Overload for 32 nodes.

As we can see from the above graph, the normalized routing overloads of DSDV are the smallest than the others and TORA has the highest normalized routing overload. The normalized routing overloads for AODV, TORA and DSR varied frequently for different number of nodes scenarios and with the change of Mobility Movement. But DSDV's normalized routing load varied a little in any scenario and speed.

\subsection{End to End Delay}

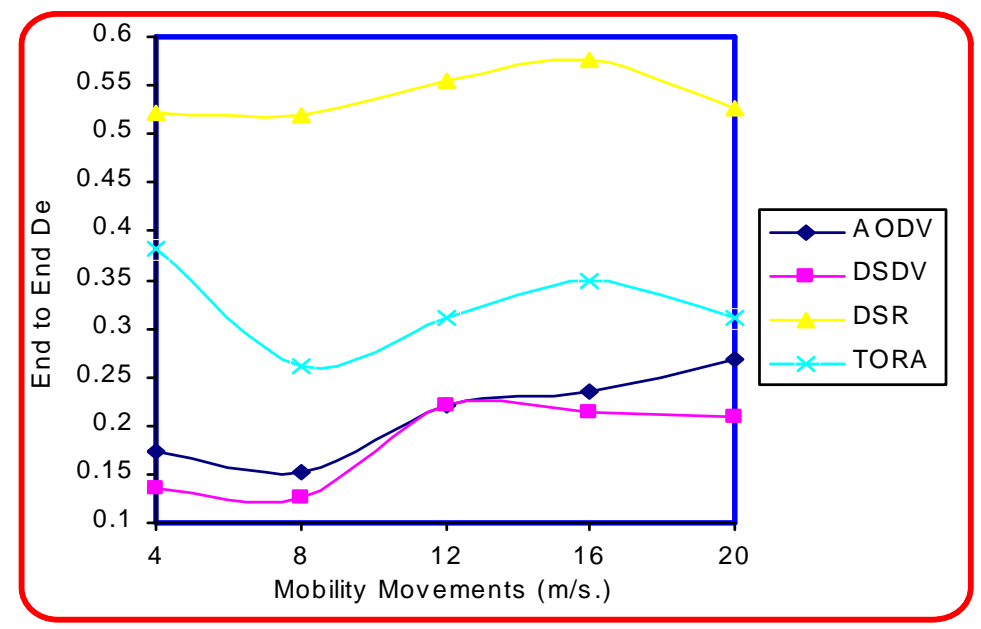

Figure 5. Average End to End Delay for 32 nodes

In the figure-5 page we see that DSDV has the shortest end-to-end delay than DSR, AODV and TORA and DSR has the highest end to end delay. In route discovery DSR needs more time as their route discovery takes more time since every intermediate node tries to extract information before forwarding the reply. Hence it slows down the transmission of packets which results of greater end-to-end delay for DSR. On the other hand DSDV is a table driven routing protocol. It needs a little end to end delay to transmit data from source to destination because the next hop address is located its table. With different number of nodes 
scenarios we have also observed that as the number of nodes increased, their end to end delays are also increased.

\subsection{Throughput}

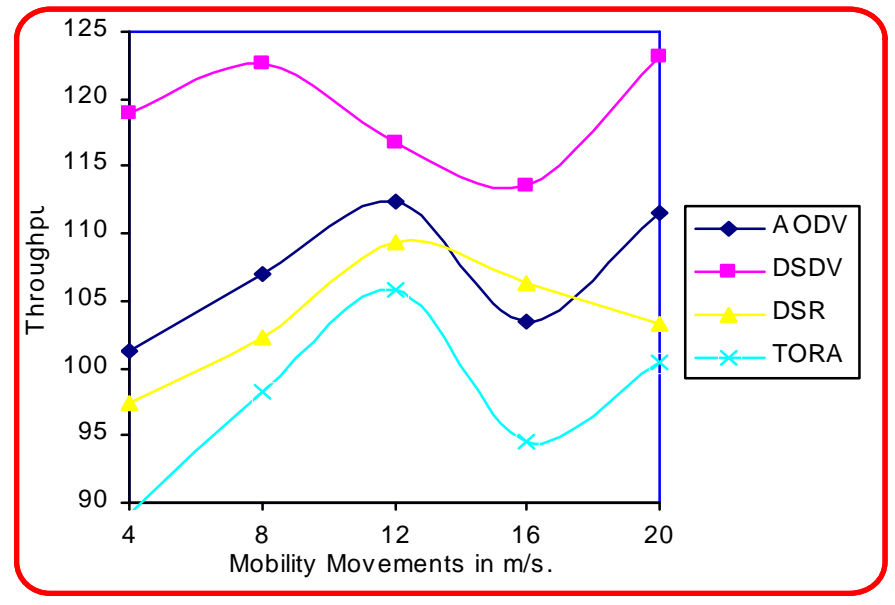

Figure 6. Throughputs for 32 nodes

Throughputs of all routing, protocols plotted in the above graph, are varying with the change of mobility movements. DSDV shows the highest performance. In all the cases TORA shows the lowest performance. The throughputs of AODV are marginal.

\subsection{Other Observations}

\subsubsection{Data Loss}

Data loss is calculated by subtracting the number of received packets by destination nodes from the number of sending packets from the source nodes. At the time of analysis we have observed that DSR has least amount of data loss and AODV has the most amount of data loss under TCP. Here data is considered as data packets.

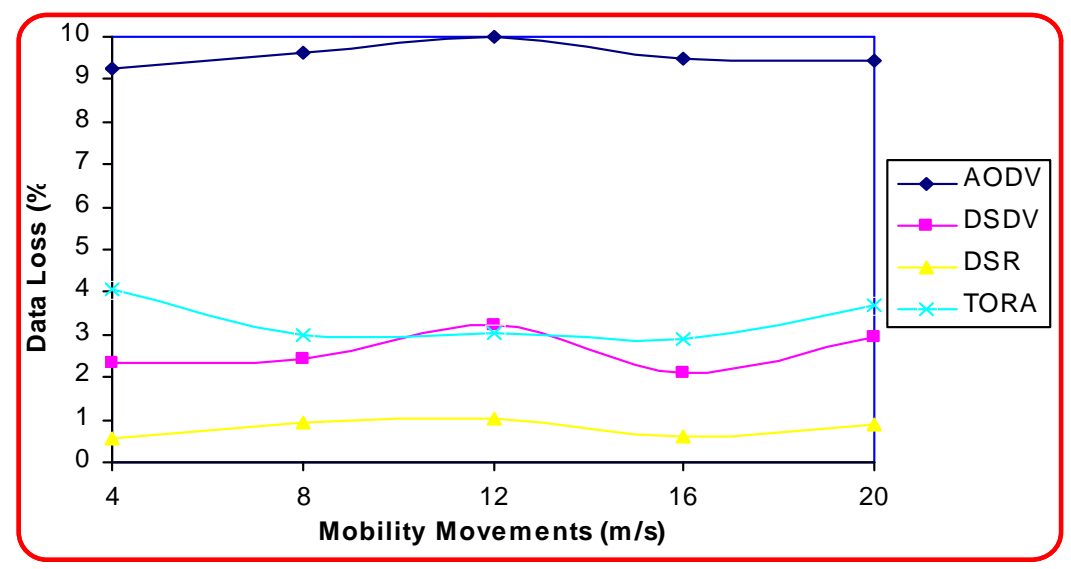

Figure 7: Data Loss for 16 nodes 


\subsubsection{Overhead in Routing Protocols}

As can be seen from figure-8, DSDV's performance is the best and AODV has the lowest performance under TCP. Here the number of nodes are 16 and mobility movement speeds are 4 $\mathrm{m} / \mathrm{s}, 8 \mathrm{~m} / \mathrm{s}, 12 \mathrm{~m} / \mathrm{s}, 16 \mathrm{~m} / \mathrm{s}$ and $20 \mathrm{~m} / \mathrm{s}$ respectively.

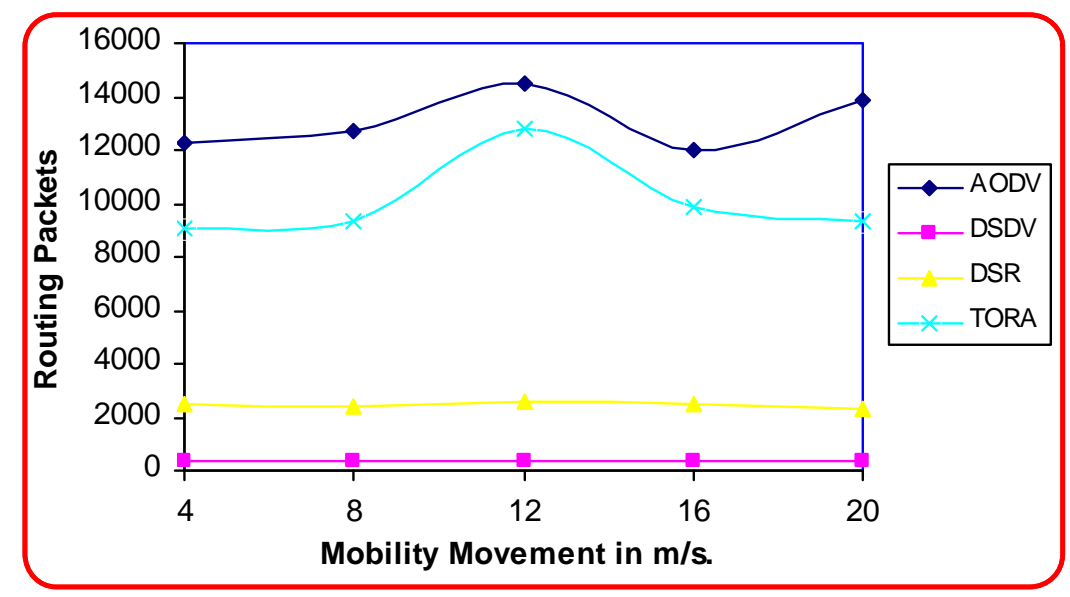

Figure 8. Overhead in Routing Protocols for 16 nodes

\section{Conclusion}

This paper makes contributions in two areas. Firstly, in this paper we have compared the performance of proactive Destination Sequenced Distance - Vector routing protocol; reactive ad hoc on demand distance vector protocol, Dynamic Source Routing protocol and reactive temporary ordered routing algorithm protocol in mobile ad hoc networks under ftp traffic. Secondly, we have presented the comprehensive results of packet delivery fraction, normalized routing overload, end to end delay, throughput and data loss over mobile ad hoc networks of thirty two mobile nodes moving about and communicating with each other at varying time. We have already discussed regarding the performances of our concern routing protocols on different performance metrics in the previous section. Considering all the performance metrics we can conclude that the DSDV shows better performance on TCP than other discussed routing protocols.

In our performance analysis we have considered only the unicast routing and various security issues are also out of the scope of this paper.

\section{REFERENCES:}

[1] C. S. R. Murthy and B. S. Manoj, Ad Hoc Wireless Networks, Architectures and Protocols, 2nd ed., Pearson Education, 2008.

[2] C. E. Perkins and P. Bhagwat, Highly Dynamic Destination- Sequenced Distance Vector Routing, ACM SIGCOMM Computer Commun. Rev., Oct. 1994, pp. 234-244.

[3] Charles E. Parkins, Mobile Ad-hoc Network Terminology, Internet-draft, draft-ietf- term00.text, March,1997.

[4] David B. Jhonson and David A. Maltz, Dynamic Source Routing in Wireless Ad Hoc Networks, in mobile computing edited by Tomasz Imielinski and Hank Korth, chapter 5, pages 153-181. Kluwer Academic Publishers. 
[5] Vincent D. Park and M. Scott Corsen, Temporary Ordered Routing Algorithm, Version 1: Functional Specification, Internet-draft, draft-ietf-manet-tora-spec-01.text, August-1998.

[6] N. Qasim, F. Said and H. Aghvami, Mobile Ad Hoc Networking Protocols' Evaluation through Simulation for Quality of Service, IAENG International Journal of Computer Science, 36:1, IJCS_36_1_10, Advance online publication: 17 February 2009

[7] Scott Corson and Joseph Macker, Mobile Ad-hoc Networking (MANET): Routing Protocol Performance Issues and Evaluation Considerations, Internet-draft, draft-ietf-manet-issues-01.text, March, 1998.

[8] C.E. Perkins and E.M. Royer, Ad-Hoc On-Demand Distance Vector Routing, Proceedings of IEEE WMCSA'99, New Orleans, LA, Feb. 1999, pp. 90-100.

[9] S. Murthy, and J. J. Garcia-Luna-Aceves, An Efficient Routing Protocol for Wireless Networks, ACM Mobile Networks and Applications Journal, Special Issue on Routing in Mobile Communication Networks, vol. 1, no. 2, Oct. 1996, pp. 183-197.

[10] C.E. Perkins and E.M. Royer, Ad-Hoc On-Demand Distance Vector Routing, Proceedings of IEEE WMCSA'99, New Orleans, LA, Feb. 1999, pp. 90-100. 